\title{
Gram stain-guided antibiotic choice: a GRACEful method to safely restrict overuse of broad-spectrum antibiotic agents
}

\author{
Jumpei Yoshimura*, Kazuma Yamakawa, Takahiro Kinoshita and Satoshi Fujimi
}

The rapid pandemic spread of multidrug-resistant (MDR) pathogens and the paucity of new, effective antibiotics are placing patients' safety at risk worldwide [1]. The World Health Organization (WHO) adopted a global action plan on antimicrobial resistance, emphasising the need to optimise the use of antibiotic agents [2]. We recently reported in Critical Care the effectiveness of Gram stain results to reduce the use of broad-spectrum antibiotics [3]. Here, we would like to report the prospective validation of the usefulness of Gram staining for antibiotic choice.

We conducted a prospective observational study from July 2016 to June 2017. Patients diagnosed as having ventilator-associated pneumonia (VAP), defined by a modified clinical pulmonary infection score $\geq 5$, were enrolled and treated according to a Gram stain-guided antibiotic choice algorithm (Fig. 1). The primary outcome was clinical response of VAP (Additional file 1: Table S1).

Nineteen patients with a median age of 65 (49-81) years were enrolled during the study period. Clinical risk factors for MDR pathogens were present in 13 (68.4\%) of the VAP patients. Pathogens isolated from endotracheal aspirates are presented in Additional file 2: Table S2. The primary outcome of the clinical response rate of VAP was $68.4 \%$, which was comparable to previous trials using broad-spectrum antibiotics in similar clinical settings
(Additional file 3: Table S3). Treatment failure occurred in six patients: antibiotic therapies were continued for more than 14 days in three patients, and pneumonia relapsed within 7 days after the end of therapy in the other three patients. The administered antibiotics did not cover pathogens isolated from an endotracheal aspirate in one patient (5.3\%). The algorithm proposed narrower-spectrum antibiotics in 15 patients $(78.9 \%)$ than those proposed by the 2016 Infectious Diseases Society of America-American Thoracic Society VAP guidelines [4] (Fig. 2). We restricted the use of anti-methicillin-resistant Staphylococcus aureus agents and anti-pseudomonal agents in 12 and 10 patients, respectively.

Our Gram stain-guided antibiotic choice algorithm was shown not only to safely guide appropriate initial antibiotic therapy but also to properly cure VAP. On the basis of the promising results of this study, we are conducting a multicentre, randomised, non-inferiority trial (GRam stain-guided Antibiotics ChoicE for Ventilator-Associated Pneumonia (GRACE-VAP)) to compare our Gram stain-guided treatment with guidelines-based treatment for patients with VAP (ClinicalTrials.gov NCT03506113, registered on 29 March 2018) [5]. Because Gram staining is an inexpensive examination and is easy to perform worldwide, including in developing countries, it could be a GRACEful method to optimise the use of antibiotics safely throughout the world.

* Correspondence: jumpei.y0210@gmail.com

Division of Trauma and Surgical Critical Care, Osaka General Medical Center, 3-1-56 Bandai-Higashi, Sumiyoshi, Osaka 558-8558, Japan 


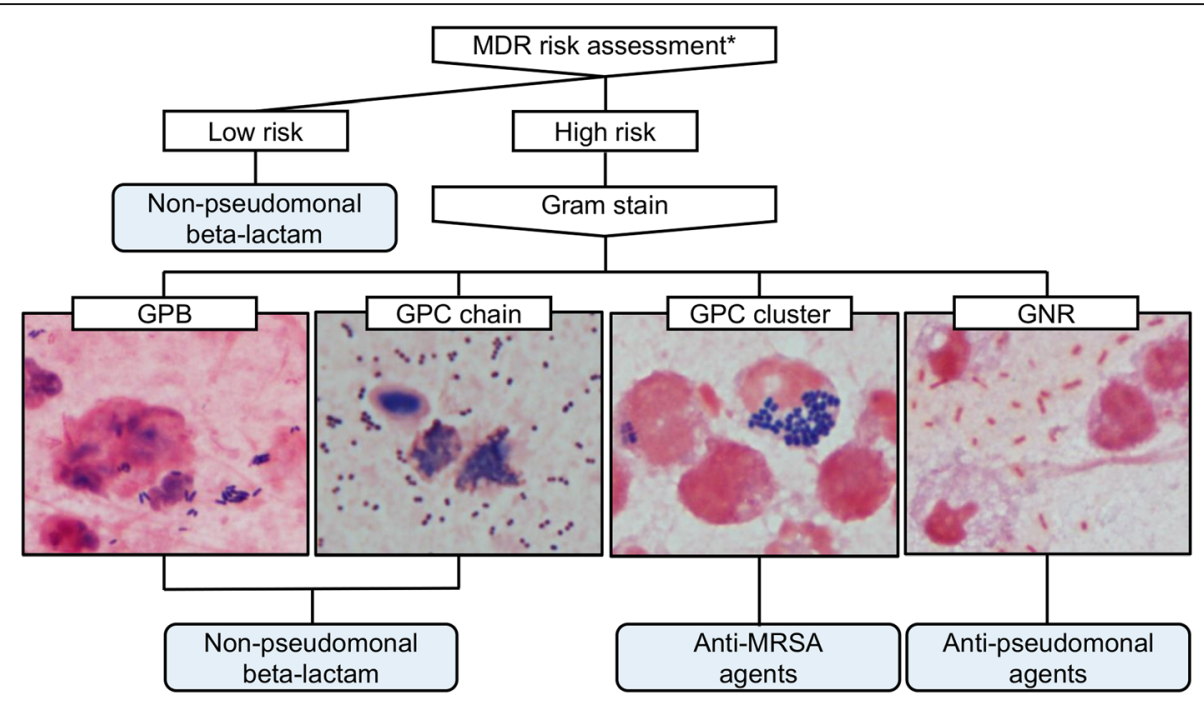

The combination of an anti-pseudomonal agent and an anti-MRSA agent is selected when the Gram stain results show both GPC clusters and GNR.

Fig. 1 Algorithm of Gram stain-guided antibiotic choice. *Patients with any of the following conditions considered at high risk: antimicrobial therapy in preceding 90 days, hospital stay of $96 \mathrm{~h}$ or longer, chronic dialysis, immunosuppressive disease or therapy, nursing home admission, septic shock at time of ventilator-associated pneumonia or colonising MDR pathogens. GPB Gram-positive bacilli, GPC Gram-positive cocci, GNR Gram-negative rods, MDR multidrug-resistant, MRSA methicillin-resistant Staphylococcus aureus

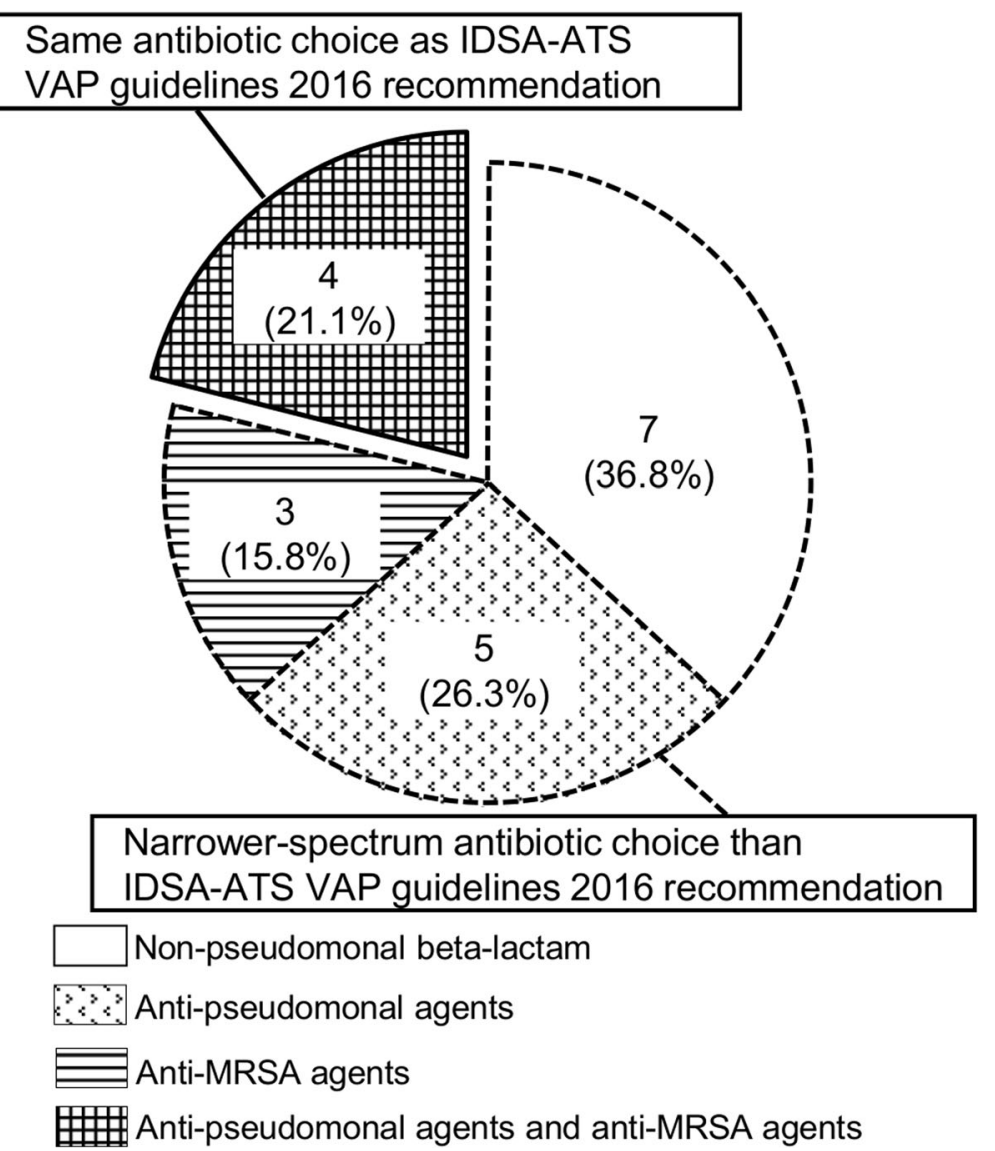

Fig. 2 Details of administered antibiotic agents. IDSA-ATS Infectious Diseases Society of America-American Thoracic Society, MRSA methicillinresistant Staphylococcus aureus, VAP ventilator-associated pneumonia 


\section{Additional files}

Additional file 1: Table S1. Definition of clinical response of ventilatorassociated pneumonia. (DOCX $21 \mathrm{~kb})$

Additional file 2: Table S2. Pathogens associated with ventilatorassociated pneumonia. MRSA: methicillin-resistant Staphylococcus aureus. (DOCX $22 \mathrm{~kb}$ )

Additional file 3: Table S3. Clinical response in the present study and previous studies. VAP: ventilator-associated pneumonia; HAP: hospital-acquired pneumonia. (DOCX $22 \mathrm{~kb}$ )

\section{Abbreviations}

MDR: Multidrug resistant; VAP: Ventilator-associated pneumonia; WHO: World Health Organization

\section{Acknowledgements}

The authors would like to thank Dr Morimoto, Dr Ohta and Dr Nakamura for thier GRACEful effort in our research.

\section{Funding}

The authors received no financial support for the study.

\section{Availability of data and materials}

The datasets used and analysed during the current study are available from the corresponding author on reasonable request.

\section{Authors' contributions}

JY conceived and designed this study, performed data acquisition and analyses, and was responsible for drafting, editing and submission of the manuscript. KY exerted a major impact on the interpretation of data. TK critically contributed to the design of the study and participated in data collection and interpretation. SF exerted a major impact on the interpretation of data and critical appraisal of the manuscript. All authors read and approved the final manuscript.

\section{Ethics approval and consent to participate}

This study was approved by the Ethics Committee of Osaka General Medical Center. All participants consented prior to enrolment.

\section{Consent for publication}

Not applicable.

\section{Competing interests}

The authors declare that they have no competing interests.

\section{Publisher's Note}

Springer Nature remains neutral with regard to jurisdictional claims in published maps and institutional affiliations.

Received: 12 November 2018 Accepted: 21 November 2018

Published online: 14 December 2018

\section{References}

1. Laxminarayan R, Duse A, Wattal C, Zaidi AK, Wertheim HF, Sumpradit N, et al. Antibiotic resistance - the need for global solutions. Lancet Infect Dis. 2013;12: 1057-98.

2. WHO. Global action plan on antimicrobial resistance. 2015. http://apps.who.int/ iris/bitstream/10665/193736/1/9789241509763_eng.pdf. Acsessed 1 Nov 2018.

3. Yoshimura J, Kinoshita T, Yamakawa K, Matsushima A, Nakamoto N, Hamasaki T, et al. Impact of Gram stain results on initial treatment selection in patients with ventilator-associated pneumonia: a retrospective analysis of two treatment algorithms. Crit Care. 2017;21:156.

4. Kalil AC, Metersky ML, Klompas M, Muscedere J, Sweeney DA, Palmer LB, et al. Management of Adults With Hospital-acquired and Ventilator-associated Pneumonia: 2016 Clinical Practice Guidelines by the Infectious Diseases Society of America and the American Thoracic Society. Clin Infect Dis. 2016;63:e61-e111.

5. Yoshimura J, Yamakawa K, Kinoshita T, Ohta Y, Morimoto T. GRam stainguided Antibiotics ChoicE for Ventilator-Associated Pneumonia (GRACE-VAP) trial: rationale and study protocol for a randomised controlled trial. Trials. 2018;19:614 